\title{
Abordaje de paciente con enfermedad pulmonar obstructiva crónica en farmacia comunitaria rural
}

\section{María Guadalupe González Pérez'1, Lydia Chaparro León²}

1. Licenciada en Farmacia. Farmacéutica Comunitaria en Peñalver (Guadalajara). 2. Licenciada en Farmacia. Responsable de Servicios Profesionales Farmacéuticos Asistenciales del Colegio Oficial de Farmacéuticos de Guadalajara.

\section{PALABRAS CLAVE}

Enfermedad pulmonar obstructiva crónica (EPOC), seguimiento farmacoterapéutico, dispositivos de inhalación, farmacovigilancia

\section{KEYWORDS}

Chronic obstructive pulmonary disease (copd), pharmacotherapy follow-up, inhalation devices, pharmacovigilance

\section{Razón de consulta}

Paciente de 77 años que en octubre de 2015 acude a farmacia comunitaria con fiebre, tos y mucosidad. Se deriva a su médico de atención primaria y tras derivación al hospital ingresa con diagnóstico de neumonía.

Acude a la farmacia pasado aproximadamente un mes (noviembre de 2015), refiriendo falta de apetito, nerviosismo, insomnio y molestias gástricas (dolor de estómago y náuseas).

Se oferta el servicio de Seguimiento Farmacoterapéutico (SFT) (1) y abordaje específico de EPOC usando como metodología el programa EPOCA (2) para el seguimiento específico de su enfermedad respiratoria.

\section{Exposición del caso}

- Fecha de nacimiento: 7/11/1937.

- Sexo: Varón.

- No alergias conocidas.

- Deterioro de intercambio de gases con dependencia de oxígeno domiciliario 16 horas diarias.

- Peso: 61,6 kg.

- Talla: $158 \mathrm{~cm}$.

- IMC: $24,67 \mathrm{~kg} / \mathrm{m}^{2}$.

- Presión arterial: 110/60 mmHg.

- Frecuencia cardiaca: $92 \mathrm{lpm}$.

- Glucemia basal: $113 \mathrm{mg} / \mathrm{ml}$.

- Insuficiencia renal leve corregida.

- Hábito tabáquico: exfumador desde hace 3 años (2-3 paquetes al día durante 60 años).

- Hábitos enólicos.

- Vacunado de la gripe y neumococo.

Los problemas de salud y medicación asociada del paciente se pueden consultar en el estado de situación (figura 3). Cabe destacar como patologías asociadas Enfermedad Pulmonar Obstructiva Crónica (EPOC) e insuficiencia cardiaca. Como medicación relevante cuenta con levofloxacino, como tratamiento esporádico por el cuadro reciente de neumonía, y el uso de diferentes inhaladores tanto de mantenimiento como de rescate.

Mejor comunicación de Servicios Profesionales Farmacéuticos (SPF). Premio 'EPOCA' a la mejor comunicación de SPF en prevención y control de la EPOC. VIII Congreso Nacional de Farmacéuticos Comunitarios celebrado en Alicante del 24 al 26 de mayo de 2018.

Financiación: ninguna.

Conflicto de intereses: ninguno.

Cite este artículo como: González MG, Chaparro L. Abordaje de paciente con enfermedad pulmonar obstructiva crónica en farmacia comunitaria rural. Farmacéuticos Comunitarios. 2019 Mar 29; 11(1):19-23. doi:10.5672/FC.2173-9218.(2019/ Vol11).001.04

Correspondencia: Maria Guadalupe González Pérez (lupegope@gmail.com).

ISSN 1885-8619 @SEFAC (Sociedad Española de Farmacia Familiar y Comunitaria). Todos los derechos reservados. 


\section{Intervención}

Lo primero que nos planteamos es abordar la EPOC siguiendo la metodología del programa EPOCA.

\section{El manejo de los inhaladores prescritos}

Se valora la técnica de inhalación usando el checklist consensuado por semFYC, SEMERGEN, SEMG y SEPAR, evaluando el número de errores presentes en el uso de los diferentes inhaladores (figura 1a-c):

- Spiriva 18 mcg 30 cápsulas inhalador Handihaler.

- Seretide Accuhaler 50/500 mcg polvo inhalador.

- Ventolin 100 mcg/pulsación aerosol.

No disponemos de Incheck dial G16 para conocer el flujo inspiratorio, pero sí constatamos con placebos sonoros que tiene flujo inspiratorio suficiente pero no tiene buena coordinación motora.

Se registra la adherencia a través del test de Morisky Green

¿Se olvida de tomar alguna vez los medicamentos inhalados? No.

¿Toma los inhaladores a la hora indicada? Sí.

¿Deja alguna vez de tomarlos? No.

Si alguna vez le sienta mal ¿deja de tomar la medicación? No.

El paciente es adherente según test en todas las visitas.

\section{Conocimiento de su enfermedad}

Paciente que desconoce en qué consiste su enfermedad, la evolución y cómo actúan los medicamentos. Se trabaja con él el conocimiento de la cronicidad de la patología y la importancia de realizar correctamente la técnica de inhalación para cada dispositivo.

Se hace especial hincapié en los hábitos de vida saludables y en reducción del consumo enólico.

Medición de cuestionario de calidad de vida (CAT): 27

(figura 2)

Vida muy limitada para las actividades domésticas diarias con impedimentos para descansar y seguir con sus relaciones sociales habituales.
Además del abordaje de la EPOC, realizamos Seguimiento Farmacoterapéutico (SFT) (1) (3) (figura 3).

1. Detectamos probabilidad de Reacción Adversa al Medicamento (RAM): Roflumilast: entre sus reacciones adversas encontramos astenia, ansiedad, dolor abdominal, nerviosismo... referidas por el paciente en la entrevista inicial.

2. Interacción de Levofloxacino con Ivabradina:

La administración conjunta de fármacos que prolonguen el intervalo QT con Ivabradina podría dar lugar a la aparición de arritmias cardiacas graves.

3. Interacción de Levofloxacino con Salmeterol:

La administración conjunta de fármacos que prolongan el intervalo QT con agonistas beta-adrenérgicos podría dar lugar a la aparición de arritmias cardiacas graves.

Una vez detectados los problemas relacionados con los medicamentos (PRM) se realizan las siguientes intervenciones:

- Facilitar información personalizada del medicamento.

- Notificar a farmacovigilancia de acuerdo a la legislación vigente (4).

- Derivar al médico comunicando PRM/RNM y proponiendo cambios en el tratamiento:

1. Cambiar Levofloxacino por Cefditoreno.

2. Roflumilast es para el tratamiento de mantenimiento de la EPOC grave con exacerbaciones frecuentes. Entre las RAM más comunes: molestias gástricas, dolor abdominal, insomnio y astenia. Se propone introducir un protector gástrico, el cual podría disminuir estos síntomas. Valorar la introducción de ansiolítico por si el paciente refiere nerviosismo e incapacidad de conciliar el sueño. En cuanto a la astenia se propone la toma de una ampolla de astenolit 1 vez al día durante 2 meses pues el tratamiento con roflumilast es esporádico.

\section{Resultados}

El médico realiza los cambios de medicación propuestos:

\section{Nueva medicación}

- Cefditoreno 400 mg 10 comp, 1-0-0

- Lorazepam $1 \mathrm{mg} 50$ comp, 0-0-1
- Omeprazol 20 mg 28 comp, 1-0-0

- Astenolit ampollas bebibles, 1-0-0

Tras un mes el paciente refiere una mejoría en su apetito y estar un poco más tranquilo.

El paciente es adherente pero la técnica de inhalación con cartucho presurizado no se consigue realizar correctamente por la incapacidad de coordinar. Se manda carta de derivación al médico explicando este hecho y se sustituye Ventolin por el Terbasmin 500 mcg turbuhaler, al no poder sincronizarse bien.

Los demás inhaladores los controla perfectamente.

Ha adquirido hábitos saludables y es consciente de su propio autocuidado.

A los 3 meses desde su inicio se le retiró lorazepam y roflumilast.

Continúa con omeprazol.

Desde el último ingreso, hace 1 año y 3 meses, no ha hecho ninguna exacerbación.

Nota: Este paciente estuvo en SFT y control de EPOC en farmacia comunitaria desde noviembre de 2015 hasta ingreso en vivienda tutelada en febrero de 2017. En el caso que nos ocupa, según informe de alta de hospitalización en neumología (4/12/2017), cabe destacar lo que escribe el neumólogo literalmente: "Llaman la atención tantas agudizaciones y disnea de pequeños esfuerzos. En 2015 múltiples agudizaciones, en 2016 estable y en 2017 nuevamente cinco agudizaciones".

Nuestro trabajo de campo se realizó durante el año 2016.

\section{Conclusiones}

La motivación y empoderamiento del paciente es esencial para el control y manejo de una patología crónica como es la EPOC tan susceptible de sufrir exacerbaciones que limitan la calidad de vida del paciente.

El autocuidado, el trabajo constante de la técnica de inhalación y el fomento de hábitos de vida saludables transmitidos desde la farmacia comunitaria son esenciales para controlar una enfermedad respiratoria tan compleja como la EPOC.

El trabajo multidisciplinar con todos los agentes sanitarios implicados es imprescindible para conseguir resultados positivos en salud. 


\section{Aerolizer $^{\circledR}$, Handihaler ${ }^{\circledR}$, Breezhaler ${ }^{\circledR}$}

\begin{tabular}{|c|c|c|}
\hline Visita inicial & $2{ }^{a}$ visita & 3. ${ }^{a}$ visita \\
\hline 区Sí $\square$ No & 区Sí $\square$ No & 区 Sí $\square$ No \\
\hline Sí $\square$ No & 区Sí $\square$ No & 区 Sí $\square$ No \\
\hline$\square$ Sí No & $\square$ Sí $\square$ No & X Sí $\square$ No \\
\hline SSí $\square$ No & 政 $\square$ No & ¿ Sí $\square$ No \\
\hline$\square$ Sí No & $\square$ Sí $\mathbb{R}$ No & Q Sí $\square$ No \\
\hline$\star$ Sí $\square$ No & 区Sí $\square$ No & Sí $\square$ No \\
\hline 区 Sí $\square$ No & 区 Sí $\square$ No & 远 Sí $\square$ No \\
\hline$\square$ Sí No & 政 $\square$ No & 准 $\square$ No \\
\hline TSí $\square$ No & Sí $\square$ No & $\square$ Sí No \\
\hline$\square$ Sí No & $\square$ Sí No & KSí $\square$ No \\
\hline 区 Sí $\square$ No & Q Sí $\square$ No & ⿷匚Sí $\square$ No \\
\hline דSí $\square$ No & EXSí $\square$ No & $\triangle$ Sí $\square$ No \\
\hline ¿ Sí $\square$ No & $\triangle$ Sí $\square$ No & ¿ Sí $\square$ No \\
\hline
\end{tabular}

Figura 1a Valoración del uso de diferentes inhaladores por el paciente (Spiriva 18 mcg 30 cápsulas inhalador Handihaler)

\section{Accuhaler $^{\circledR}$}

1. ¿Abre correctamente el dispositivo? (deslizando el protector apoyando el dedo pulgar en la hendidura)

2. ¿Carga el dispositivo hasta oir un clic?

3. ¿Pone el disco paralelo al suelo?

4. ¿Realiza una espiración previa, evitando hacerlo sobre el dispositivo o en el dispositivo?

5. ¿Acopla correctamente la boca a la boquilla?

6. ¿Realiza una inspiración enérgica y profunda de forma constante?

7. ¿Aguanta la respiración 8-10 segundos?

8. ¿Expulsa el aire lentamente por la nariz o por la boca?

9. ¿Espera al menos 30 segundos antes de repetir los pasos del 1 al 8 ? (cerrando el dispositivo al principio)

10. ¿Cierra correctamente el dispositivo?

11 ¿Se enjuaga la boca con agua al finalizar? (registrar pero no puntuarlo como error de la técnica)

12. ¿Sabe cuándo se ha acabado el producto (lectura de las dosis del aparato)?

Visita inicial

2. $^{\text {a }}$ visita

3. ${ }^{a}$ visita

¿ Sí $\square$ No

ه Sí $\square$ No

¿ Sí $\square$ No

$\square$ Sí [No

ESí $\square$ No

$\square$ Sí No

$\checkmark$ Sí $\square$ No

¿ Sí $\square$ No

¿ Sí $\square$ No

도í $\square$ No

$\square$ Sí

凶Sí $\square$ No

就 $\square$ No

⿷Sí $\square$ No

田 Sí $\square$ No

$\square$ Sí

$\square$ Sí No

\Sí $\square$ No

$\square$ Sí

Sí $\square$ No

ㄴí $\square$ No

¿ Sí $\square$ No

¿Sí $\square$ No
匹Sí $\square$ No

$\triangle$ Sí $\square$ No

Ð Sí $\square$ No

[TSí $\square$ No

㨁Sí $\square$ No

¿Sí $\square$ No

¿ Sí $\square$ No

Sí $\square$ No

KSí $\square$ No

任 $\square$ No

$\triangle$ Sí $\square$ No

Sí $\square$ No

Figura 1b Valoración del uso de diferentes inhaladores por el paciente (Seretide Accuhaler 50/500 mcg polvo inhalador) 


\section{Inhalador de cartucho presurizado (MDI)}

\begin{tabular}{|c|c|c|}
\hline Visita inicial & $2^{a}$ visita & 3. ${ }^{a}$ visita \\
\hline$\triangle S_{1} \square$ No & $\nabla$ Sí $\square$ No & 区 Sí $\square$ No \\
\hline 口 Sí $\mathbf{E}$ No & $\square$ Sí $\square$ No & $\square$ Sí $\square$ No \\
\hline$\triangle$ Sí $\square$ No & $\triangle$ Sí $\square$ No & 因 Sí $\square$ No \\
\hline$\square$ Sí $\square$ No & $\triangle$ Sí $\square$ No & ॠSí $\square$ No \\
\hline$\triangle$ Sí $\square$ No & Sí $\square$ No & $\triangle$ Sí $\square$ No \\
\hline$\square$ Sí ENo & $\square$ Sí No & $\square$ Sí No \\
\hline 口 Sí $\square$ No & $\square$ Sí $\mathbb{N}$ No & $\square$ Sí $\mathbb{Q}$ No \\
\hline$\triangle$ Sí $\square$ No & Sí $\square$ No & $\square$ Sí $\square$ No \\
\hline$\square$ Sí No & 区 Sí $\square$ No & $\triangle$ Sí $\square \mathrm{N}$ \\
\hline$\square$ Sí EINo & $\square$ Sí $₫$ No & ¿ Sí $\square$ No \\
\hline$\triangle$ Sí $\square$ No & 区Sí $\square$ No & 凶Sí $\square$ No \\
\hline$\square$ Sí & Sí $\square$ No & ESí $\square$ No \\
\hline$\square$ Sí $\square$ No & $\square$ Sí No & $\square$ Sí $\triangle$ No \\
\hline
\end{tabular}

Figura 1c Valoración del uso de diferentes inhaladores por el paciente (Ventolin 100 mcg/pulsación aerosol)

\begin{tabular}{|c|c|c|c|c|c|}
\hline Estoy muy contento & 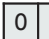 & 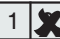 & 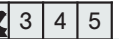 & Estoy muy triste & Puntuación \\
\hline Nunca toso & 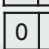 & \begin{tabular}{l|l}
1 & 2 \\
\end{tabular} & \begin{tabular}{|l|l|l|}
3 & $\times 5$ \\
\end{tabular} & Siempre estoy tosiendo & 4 \\
\hline No tengo flemas (mucosidad) en el pecho & \begin{tabular}{|l|l|l}
0 & \\
\end{tabular} & \begin{tabular}{l|l}
1 & 2 \\
\end{tabular} & \begin{tabular}{|l|l|l|}
$\mathbf{x}$ & 4 & 5 \\
\end{tabular} & Tengo el pecho completamente cargado de flema (mucosidad) & 3 \\
\hline $\begin{array}{l}\text { Cuando subo una pendiente o un tramo } \\
\text { de escaleras, no me falta aire }\end{array}$ & \begin{tabular}{|l|l|l|l|}
0 & 1 \\
\end{tabular} & \begin{tabular}{l|l|}
1 & 2 \\
\end{tabular} & \begin{tabular}{|l|l|l|}
3 & 4 & $\mathbf{8}$ \\
\end{tabular} & $\begin{array}{l}\text { Cuando subo una pendiente o un tramo de } \\
\text { escaleras, me falta aire siempre }\end{array}$ & 5 \\
\hline $\begin{array}{l}\text { No me siento limitado para } \\
\text { realizar actividades domésticas }\end{array}$ & \begin{tabular}{|l|l|l|l|l}
0 & 0 \\
\end{tabular} & \begin{tabular}{l|l|}
1 & 2 \\
\end{tabular} & \begin{tabular}{|l|l|l|}
$\mathbf{X}$ & 4 & 5 \\
\end{tabular} & Me siento muy limitado para realizar actividades domésticas & 3 \\
\hline $\begin{array}{l}\text { Me siento seguro al salir de casa, a pesar } \\
\text { de la afección pulmonar que padezco }\end{array}$ & \begin{tabular}{|l|l|l|l}
0 & \\
\end{tabular} & \begin{tabular}{l|l|}
1 & 2 \\
\end{tabular} & \begin{tabular}{|l|l|l|}
3 & $\mathbf{X}$ & 5 \\
\end{tabular} & $\begin{array}{l}\text { No me siento nada seguro al salir de casa, } \\
\text { debido a la afección pulmonar que padezco }\end{array}$ & 4 \\
\hline Duermo sin problemas & & \begin{tabular}{l|l|}
1 & 2 \\
\end{tabular} & \begin{tabular}{|l|l|l|}
3 & $\mathbf{x}$ & 5 \\
\end{tabular} & $\begin{array}{l}\text { Tengo problemas para dormir debido a la afección pulmonar } \\
\text { que padezco }\end{array}$ & 4 \\
\hline Tengo mucha energía & 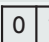 & \begin{tabular}{l|l|l}
1 & 2
\end{tabular} & \begin{tabular}{|l|l|l|}
3 & $\times 5$ \\
\end{tabular} & No tengo ninguna energía & 27 \\
\hline
\end{tabular}

Figura 2 Cuestionario de calidad de vida (CAT)

Cuestionario que sirve para medir el impacto que tiene la EPOC en el bienestar y la vida diaria del paciente. Es un cuestionario breve que valora la presencia de tos, flema, opresión torácica, disnea de esfuerzo, limitación para las actividades domésticas, inseguridad para salir de casa, calidad del sueño y percepción de la "energía" en el paciente. A mayor puntuación, peor estado de salud (5). 


\begin{tabular}{|c|c|c|c|c|c|c|c|c|c|c|c|c|c|}
\hline $\begin{array}{l}\text { Fecha de } \\
\text { inicio }\end{array}$ & $\begin{array}{l}\text { Problema de } \\
\text { Salud }\end{array}$ & control & $\begin{array}{l}\text { Fectra de } \\
\text { inicio }\end{array}$ & $\begin{array}{l}\text { Código } \\
\text { Nacioral }\end{array}$ & $\begin{array}{c}\text { Principio activo y dosis por } \\
\text { toma }\end{array}$ & $\begin{array}{l}\text { Pautre } \\
\text { prescrite }\end{array}$ & $\begin{array}{l}\text { Pauta } \\
\text { utilizada }\end{array}$ & PRM & $\mathrm{N}$ & $\mathrm{E}$ & $s$ & & SM \\
\hline \multirow{5}{*}{2000} & \multirow{5}{*}{ EPOC } & \multirow{5}{*}{$\begin{array}{l}\Gamma \text { no } \\
\Gamma \text { sí } \\
\Gamma \text { nosabe }\end{array}$} & \multirow{5}{*}{$\begin{array}{l}2003 \\
20013\end{array}$} & 64832 & TIOTFOPIO18 MKG & $1-0.0$ & $1-0.0$ & \multirow{5}{*}{$\begin{array}{l}\text { RAM } \\
\text { POFLUMMIL } \\
\text { INTERACCIÓ } \\
\text { LEVOFLOXAC } \\
\text { +SALMETER }\end{array}$} & \multirow{5}{*}{ sI } & \multirow{5}{*}{ sI } & si & \multirow{5}{*}{$\begin{array}{l}\text { Г RNM } \\
\text { Г" Riespode } \\
\text { RNM } \\
\Gamma \text { No RNM }\end{array}$} & \multirow{5}{*}{$\begin{array}{l}\Gamma \text { No Necesidad } \\
\Gamma \text { Necesidad } \\
\Gamma \text { Inefectividad } \\
\Gamma \text { Insceu ridad }\end{array}$} \\
\hline & & & & 700539 & FLUTICASONA 50OMCG & $2-0-2$ & $2-0-2$ & & & & si & & \\
\hline & & & & 700639 & SALMETEPOL $5 O$ MCG & $20-2$ & $2-0-2$ & & & & $?$ & & \\
\hline & & & & 66845 & FOFLUMILASTSOO MKG & $1-0.0$ & $1-0.0$ & & & & N & & \\
\hline & & & & க6\%6 & SALEUTAMOL 100 MKG & Dermanda & Derranda & & & & sI & & \\
\hline \multirow{4}{*}{2001} & \multirow{4}{*}{$\begin{array}{l}\text { INEUFICIENCIA } \\
\text { CARDIACA }\end{array}$} & \multirow{4}{*}{$\begin{array}{l}\Gamma \text { No } \\
\Gamma \text { sí } \\
\Gamma \text { Nosabe }\end{array}$} & \multirow[t]{4}{*}{20012} & E 2451 & IVAERADINA 5 MG & $1-0-0$ & $1-0.0$ & \multirow{4}{*}{$\begin{array}{l}\text { INTERACCIÓ } \\
\text { LEVOFLOXAC } \\
\text { + IVABRAINA }\end{array}$} & \multirow{4}{*}{ si } & \multirow{4}{*}{ sI } & $?$ & \multirow{4}{*}{$\begin{array}{l}\Gamma \text { RNM } \\
\text { Г Riesgode } \\
\text { RNMM } \\
\Gamma \text { No RNM }\end{array}$} & \multirow{4}{*}{$\begin{array}{l}\Gamma \text { No Necesidad } \\
\Gamma \text { Necesidad } \\
\Gamma \text { Inefectividad } \\
\Gamma \text { Inscru ridad }\end{array}$} \\
\hline & & & & & & & & & & & & & \\
\hline & & & & & & & & & & & & & \\
\hline & & & & & & & & & & & & & \\
\hline \multirow{4}{*}{2001} & \multirow{4}{*}{ HTA } & \multirow{4}{*}{$\begin{array}{l}\Gamma \text { No } \\
\Gamma \text { sí } \\
\Gamma \text { nosabe }\end{array}$} & \multirow{4}{*}{2002} & & & & & & \multirow{4}{*}{ sI } & \multirow{4}{*}{ sI } & si & \multirow{4}{*}{$\begin{array}{l}\Gamma \text { RNM } \\
\Gamma \begin{array}{l}\text { Riesgode } \\
\text { RNM }\end{array} \\
\Gamma \times \text { No RNM }\end{array}$} & $\Gamma$ no Necesidad \\
\hline & & & & & & & & & & & & & $\Gamma$ Necesidad \\
\hline & & & & 670264 & RAMIPRIL 5 MG & $1-0.0$ & $1-0.0$ & & & & & & $\Gamma$ \\
\hline & & & & & & & & & & & & & $\Gamma$ Inscruridad \\
\hline & & & Nov 20015 & 693488 & LEVOFLOXACINO $500 \mathrm{MG}$ & $1-0.0$ & $1-0.0$ & INTERACCIÓ & & & $?$ & Г RNM & $\Gamma$ no Necesidad \\
\hline & & mo & Nov 20015 & $\$ 4312$ & ACETILCISTEINA $600 \mathrm{MG}$ & $1-0.0$ & $1-0.0$ & LEVOFLOXAC & & & si & & $\Gamma$ Necesidad \\
\hline Now & NEUMONIA & Гsí & & & & & & SALMETER & sI & $?$ & & $\ulcorner$ RNM & $\Gamma$ Inofoctividy \\
\hline & & Fv nosabe & & & & & & $\stackrel{e}{e}$ & & & & $\Gamma$ No RNM & 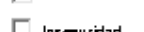 \\
\hline & & & 2001 & 157170 & HEXETIDINA & $1-1-1$ & 1-1-1 & & & & s! & F- оми & ГГ \\
\hline & & $\Gamma$ No & (- & ( & & 年 & - & & & & 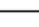 & Rinlin & \\
\hline & PERIODONIITIS & F sí & & & & & & & sl & sI & & $\Gamma$ Riesgode & 1 Necesidad \\
\hline 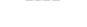 & & r Mncato & & & & & & & & & & Fivion & Г Inefectividad \\
\hline & & & & & & & & & & & & Fo No RNM & $\Gamma$ Inscauridad \\
\hline & & F no & & & & & & & & & & $\ulcorner$ RNM & $\Gamma$ no Necesidad \\
\hline $\mathrm{Nax} 2005$ & MOLESTIAS & & & & & & & & & & & Riesgode & $\Gamma$ Necesidad \\
\hline whe & GASTRICAS & Г- pecate & & & & & & & & & & RNM & $\ulcorner$ Inefectividad \\
\hline & & & & & & & & & & & & $\Gamma$ No RNM & $\Gamma_{\text {Insemuridad }}$ \\
\hline & & & & & & & & & & & & $\Gamma_{\text {RNM }}$ & $\Gamma$ no Neces idad \\
\hline 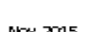 & ASTENA & $r_{s}$ & & & & & & & & & & Fiesgode & $\Gamma$ Necesidad \\
\hline 年 & ASTEDISA & 正 & & & & & & & & & & RNM & $\Gamma$ Inefectividad \\
\hline & & $\Gamma$ Nosabe & & & & & & & & & & Г No RNM & $\Gamma_{\text {Inscruridad }}$ \\
\hline & & & & & & & & & & & & $\Gamma$ RNM & $\Gamma$ no Necesidad \\
\hline & & $-a$ & & & & & & & & & - & Fiesgode & $\Gamma$ Necesidad \\
\hline Nov 20015 & NERVIOSISMO & ¿sí & & & & & & & & & - & RNM & $\Gamma$ Inefectridad \\
\hline & & $\Gamma$ nosabe & & & & & & & & & & $\Gamma$ No RNM & $\Gamma_{\text {Insceuridad }}$ \\
\hline
\end{tabular}

Figura 3 Estado de situación (23 de noviembre de 2015)

\section{Referencias bibliográficas}

1. Grupo de Expertos. Foro de Atención Farmacéutica en Farmacia Comunitaria: Guía Práctica para los Servicios de Atención Farmacéutica en la Farmacia Comunitaria. Madrid: Consejo General de Colegios Oficiales de Farmacéuticos; 2010. Disponible en https://www. portalfarma.com/inicio/serviciosprofesionales//forofarmaciacomunitaria/ Documents/ATFC_Guia\%20FOR0.pdf
2. Plaza J, Mendoza A, Calvo E, Márquez E, Quintano JA, Trigueros JA. Curso de capacitación Época. https://www.campussefac.org/epoca/inicio

3. Bot PLUS 2.0. Base de datos del Conocimiento Sanitario. Consejo General de Colegios Oficiales de Farmacéuticos 2016. Fichas técnicas de los medicamentos citados. Disponible en https:// botplusweb.portalfarma.com/botplus. aspx
4. Sistema Español de Farmacovigilancia de medicamentos de uso humano. Agencia Española de Medicamentos y Productos Sanitarios. Disponible en https://www.aemps.gob.es/vigilancia/ medicamentosUsoHumano/home.htm y https://www.notificaram.es/

5. P.W. Jones, G. Harding, P. Berry, I. Wiklund, W-H. Chen, N. Kline Leidy. Development and first validation of the COPD assessment test. 